\title{
ON OSCILLATORY ELLIPTIC EQUATIONS ON MANIFOLDS
} BY

\author{
A. BAIDER AND E. A. FELDMAN ${ }^{1}$
}

\begin{abstract}
In this note we investigate the possibility of oscillatory behavior for a second-order selfadjoint elliptic operators on noncompact Riemannian manifolds $(M, g)$. Let $A$ be such an operator which is semibounded below and symmetric on $C_{0}^{\infty}(M) \subseteq L^{2}(M, d \mu)$ where $d \mu$ is a volume element on $M$. If $\varphi$ is a $C^{\infty}$ function such that $A \varphi=\lambda \varphi$, we would naively say that $\varphi$ is oscillatory (and by extension $\lambda$ is oscillatory if it possesses such an eigenfunction $\varphi$ ) if $M-\varphi^{-1}(0)$ has an infinite number of bounded connected components. For technical reasons this is not quite adequate for a definition. However, in $\$ 1$ we give the usual definition of oscillatory which is a slight generalization of the one above. Let $\Lambda_{0}$ be the number below which this phenomenon cannot occur; $\Lambda_{0}$ is the oscillatory constant for the operator $A$. In that $A$ is semibounded and symmetric on $C_{0}^{\infty}(M) \subseteq L^{2}(M, d \mu), A$ has a Friedrichs extension. Let $\Lambda_{c}$ be the bottom of the continuous spectrum of the Friedrichs extension of $A$. Our main result is $\Lambda_{0}=\Lambda_{c}$.
\end{abstract}

This is the exact analogue of a theorem first proved by Hartman and Putnam [6], when $M$ is the real line. More recently, John Piepenbrink [7] generalized [6] to $\mathbf{R}^{n}$, requiring in the process, a technical condition (see [7, (3.1)]), which is, roughly, a restriction on the growth at infinity of the coefficients of the principal part of $A$. For example, if

$$
A=-\sum \frac{\partial}{\partial x_{i}}\left[\left(1+|x|^{2}\right)^{1 / 2}\right]^{\alpha} \frac{\partial}{\partial x_{i}}
$$

then Piepenbrink's condition (3.1) holds if and only if $\alpha<2$. In our generalization to arbitrary noncompact manifolds, no restrictions are placed upon the behavior of $A$ at large distances. More significantly, the equality $\Lambda_{0}=\Lambda_{c}$ is valid regardless of the "amount of topology" that $M$ may possess. In [7] this type of difficulty is obviated by the fact that $\mathbf{R}^{n}$ is contractible.

The proof depends upon two theorems, Theorem A and Theorem B. Theorem B is a geometric characterization of $\Lambda_{c}$ due to one of the authors in [1], while Theorem $\mathrm{A}$ is a deformation of domain argument in the spirit of [3], [8], [9]. The paper is arranged in the following manner. In $\S 1$ we give the necessary definitions, state Theorems A and B and show how the equality follows from Theorems A and B. The remainder of the paper is a proof of Theorem A. $\$ 2$ is the analytic part of the proof of Theorem A. The core of the argument is the "crushed ice" estimates of Rauch and Taylor [8], [9], with which we prove Theorem A, provided we have a fine enough "even spacing lemma". This "even spacing lemma", is the geometric-

Received by the editors May 31, 1978 and, in revised form, April 10, 1979.

AMS (MOS) subject classifications (1970). Primary 58G99, 35J20.

${ }^{1}$ This paper was partially supported by NSF grant \# MCS-77-02757. 
topological part of the argument, as well as the most technical, and is exposed in §3. An interesting aspect of this lemma, is the need to use a triangulation of the manifold, which is a good approximation to the Riemannian structure.

Finally, we would like to thank I. Chavel for reading parts of the manuscript, and making many helpful suggestions.

1. Definitions and the statements of the results. Let $M$ be a manifold possibly with boundary. Let $g$ be a Riemannian metric on $M$, and let $\mu$ be a smooth volume element on $M$, i.e., $\mu=f d v$ where $f$ is a smooth positive function, and where $d v$ is the volume element $g$ induces on $M$. For each smooth vector field $v$ on $M$, we define the smooth functions $\operatorname{div}_{\mu} v$, the divergence of $v$ with respect to $\mu$, by the formula $d\left(i_{v} \mu\right)=\left(\operatorname{div}_{\mu} v\right) \mu$. If $f$ is a $C^{\infty}$ function defined on $M$, then

$$
\operatorname{div}_{\mu} f v=f \operatorname{div}_{\mu} v+d f(v) .
$$

Finally, if $f$ is a smooth function we define the elliptic operator

$$
\Delta_{\mu} f=-\operatorname{div}_{\mu} \operatorname{grad} f
$$

which is symmetric because

$$
\int_{M} \varphi \Delta_{\mu} \psi d \mu=\int_{M} \Delta_{\mu} \varphi \psi d \mu=\int_{M} \operatorname{grad} \varphi \cdot \operatorname{grad} \psi d \mu,
$$

where $\varphi, \psi \in C_{0}^{\infty}($ Int $M)$ are smooth functions with compact support in the interior of $M$, and - is the fiberwise inner product given by $g$.

The general operator we want to consider is of the form $A=\Delta_{\mu}+c$ where $c$ is a continuous function, such that $(A \varphi, \varphi) \geqslant l(\varphi, \varphi)$ for some $l>-\infty$, where $($, denotes the inner product on $L^{2}(M, d \mu)$, and $\varphi \in C_{0}^{\infty}(M)$. Thus $A$ is semibounded and symmetric.

Let $U$ be an open set in $M$ and let $C_{0}^{\infty}(U)$ be the $C^{\infty}$ functions with compact support in $U$. We then define

$$
\lambda_{A}(U)=\inf _{\varphi \in C_{0}^{\infty}(U), \varphi \neq 0} \frac{(A \varphi, \varphi)}{(\varphi, \varphi)} .
$$

Note that if $U \subset V$, then $\lambda_{A}(U) \geqslant \lambda_{A}(V)$.

THEOREM A. Let $M_{0}$ be a compact manifold with boundary $\partial M_{0}=N$. Let $g$ be a Riemannian metric on $M_{0}$ and $d \mu$ be a volume element on $M_{0}$. Let $c$ be a continuous function on $M_{0}$, and set $A=-\operatorname{div}_{\mu} \operatorname{grad}+c$. Let $\Lambda=\lambda_{A}\left(\right.$ int $\left.M_{0}\right)$, and pick any number $l>\Lambda$. Then we can find $M_{1} \subseteq M_{0}$, a compact submanifold with boundary, and a smooth 1-parameter family of diffeomorphism $h_{t}: M_{1} \rightarrow M_{0}$ such that

(i) $\lambda_{A}$ (int $\left.M_{1}\right)>l$,

(ii) $h_{0}=$ id, $h_{1}\left(M_{1}\right)=M_{0}$,

(iii) $h_{t}\left(M_{1}\right) \subseteq h_{t^{\prime}}\left(M_{1}\right)$ if $t \leqslant t^{\prime}$, and thus $\lambda(t)=\lambda_{A}\left(\operatorname{int}\left(h_{1-t}\left(M_{1}\right)\right)\right)$ is monotone increasing and continuous.

Let $M$ be a smooth manifold without boundary. Let $g, d \mu$ and $A$ be as above. Then $A$ defines a symmetric linear map on $C_{0}^{\infty}(M) \subseteq L^{2}(M, d \mu)$ which is semibounded below. We can then form the Friedrichs extension [10, p. 329] which we will also denote by $A$. The Friedrichs extension is a selfadjoint operator on its 
domain $D_{A}$ and possesses a spectral decomposition. Thus we can speak of the discrete and the continuous part of the spectrum. In particular, let $\lambda_{c}(A)$ denote the bottom of the continuous spectrum of $A$, i.e., $\lambda_{c}(A)=\inf \{\lambda: \lambda$ is in the continuous spectrum of $A$ \}. Baider [1] has given the following geometric characterization of $\lambda_{c}(A)$.

THEOREM B. $\lambda_{c}(A)=\sup _{K}$ compact $\lambda_{A}(M-K)$.

We will now begin to discuss oscillatory behavior. Let $M$ be a smooth manifold, $\partial M=\varnothing$. Let $g, d \mu$ and $A$ be as before. An open connected set in $M$ will be referred to as a domain. If the boundary of a domain $\Omega, \partial \Omega=\bar{\Omega}-\Omega$ is a smooth $n-1$ dimensional manifold, then we call $\Omega$ a regular domain. A domain is bounded if $\bar{\Omega}$ is compact.

Definition. Let $\lambda$ be a real number. $\Omega \subseteq M$ a domain. We call $\Omega$ a $\lambda$-domain for $A$ if $\Omega$ is bounded and if $\lambda_{A}(\Omega)=\lambda$. Notice that if $\Omega$ is a regular $\lambda$-domain (this clearly can be weakened some), then $\lambda_{A}(\Omega)$ is the lowest eigenvalue for $A$, with Dirichlet boundary data on $\partial \Omega$.

Proposition. Let $\lambda \in \mathbf{R}$. The following two statements are equivalent.

(a) For each compact subset $K \subseteq M$, we can find a regular $\lambda$-domain $\Omega$ such that $\bar{\Omega} \subseteq M-K$.

(b) For each compact subset $K \subseteq M$, we can find a $\tilde{\lambda}$-domain $\tilde{\Omega}$, such that $\overline{\tilde{\Omega}} \subseteq M-K$ and $\tilde{\lambda} \leqslant \lambda$.

Proof. It is trivial to see that (a) implies (b).

Let $M=\cup_{i=1}^{\infty} U_{i}, U_{i}$ open, regular bounded domains where $\bar{U}_{i} \subseteq U_{i+1}$. For each compact set $K$, we can find $i(K)$ such that $K \subseteq \overline{U_{i(K)}}$ and hence $M-\overline{U_{i(K)}} \subseteq$ $M-K$. If (b) holds, we can find a $\tilde{\lambda}$-domain $\Omega$ such that $\bar{\Omega} \subseteq V \subseteq M-\overline{U_{i(K)}}$, where $V$ is the connected component of $M-\overline{U_{i(K)}}$ containing $\bar{\Omega}$ and $\overline{\tilde{\lambda}}<\lambda$. If $V$ is bounded, it is therefore a regular bounded domain, such that $\partial V \cap \bar{\Omega}=\varnothing$. Thus we can find a bounded, regular domain $W, \bar{\Omega} \subseteq W \subseteq \bar{W} \subseteq V$. Therefore, $W$ is a regular $\lambda^{\prime}$-domain, $\lambda^{\prime} \leqslant \lambda$. (a) then follows from Theorem A. Finally if $V$ is not bounded, we can clearly find a regular bounded domain $W$, such that $\bar{\Omega} \subseteq W \subseteq$ $\bar{W} \subseteq V$. (a) then follows as above.

Definition. We say $\lambda$ is oscillatory for $A$ if $\lambda$ satisfies (a) or (b) of the proposition. If $\lambda$ is oscillatory and if $\lambda \leqslant \mu$, then (b) of the proposition shows that $\mu$ is also oscillatory. Thus we can define the oscillation constant $\Lambda_{0}(A)$ of $A$ to be

$$
\Lambda_{0}(A)=\inf \{\lambda: \lambda \text { is oscillatory }\} .
$$

THEOREM. $\Lambda_{c}(A)=\Lambda_{0}(A)$.

Proof. Let $M=\cup_{i=1}^{\infty} U_{i}, U_{i}$ all regular bounded domains, such that $\bar{U}_{i} \subseteq$ $U_{i+1}$. Thus $\lambda_{A}\left(M-\bar{U}_{i}\right) \leqslant \lambda_{A}\left(M-\bar{U}_{i+1}\right)$ and it follows easily from Theorem B that

$$
\Lambda_{c}(A)=\sup \lambda_{A}\left(M-\bar{U}_{i}\right)=\lim _{i \rightarrow \infty} \lambda_{A}\left(M-\bar{U}_{i}\right) .
$$

Assume $\lambda$ is oscillatory. For each $i$, we can find a regular $\lambda$-domain in $M-\bar{U}_{i}$ and 
thus a regular $\lambda$-domain in $U_{k}-\bar{U}_{i}$ for some $k>i$. Hence $\lambda_{A}\left(M-\bar{U}_{i}\right)=$ $\inf _{l>k}\left(U_{l}-\bar{U}_{i}\right) \leqslant \lambda$. which implies that $\lambda_{c}(A) \leqslant \lambda_{0}(A)$. Conversely, let $\lambda<\lambda_{0}(A)$. Choose an $\varepsilon>0$ such that $\lambda+\varepsilon<\lambda_{0}(A)$. We can then find some $U_{i}$ such that $M-\bar{U}_{i}$ contains no $\tilde{\lambda}$-domains, $\tilde{\lambda} \leqslant \lambda+\varepsilon$. Hence $\lambda_{A}\left(M-\bar{U}_{i}\right)>\lambda$ which implies $\lambda_{c}(A) \geqslant \lambda_{0}(A)$.

2. Crushed ice. For the remainder of the note let $(M, g)$ be a compact, connected $m$-dimensional Riemannian manifold and let $M_{0} \subseteq M$ be a compact $m$-dimensional manifold with $\partial M_{0}=N$. Let $d \mu$ be a volume element on $M$, let $c$ be a continuous function on $M_{0}$, let $A=-\operatorname{div}_{\mu} \operatorname{grad}+c$, and let $\Delta_{g}=-\operatorname{div}_{g} \operatorname{grad}$ be the usual Laplacian. $d \mu=f d v$ where $f>0$ and $d v$ is the Riemannian volume element. Then we can find a real number $K>0$ such that $(1 / K) d \mu<d v<K d \mu$ and let $\alpha=\inf c(x)$. If $\psi$ is a smooth function on $M_{0}$, then $\int\|\operatorname{grad} \psi\|^{2} d \mu>$ $(1 / K) \int\|\operatorname{grad} \psi\|^{2} d v$, and $\int \psi^{2} d \mu \leqslant K \int \psi^{2} d v$. Thus if $\psi$ is the first nontrivial eigenfunction for the Dirichlet problem for $M_{0} \subseteq M$, we see that

$$
\lambda_{A}\left(M_{0}\right) \geqslant \frac{1}{K^{2}} \frac{\int\|\operatorname{grad} \psi\|^{2} d v}{\int \psi^{2} d v} .
$$

Therefore, $\lambda_{A}\left(M_{0}\right) \geqslant\left(1 / K^{2}\right) \lambda_{\Delta}\left(M_{0}\right)+\alpha$, and it suffices to prove Theorem $A$ in the case where the operator $A=\Delta_{g}=\Delta$. We will denote $\lambda_{A}$ ( ) by $\lambda($ ), and furthermore denote by $B(x, r), x \in M$ and $r$ a positive real, the open metric ball centered at $x$ of radius $r$.

Proposition 2.1. Let $(M, g), M_{0} \subseteq M, \partial M_{0}=N_{0}$ be as before and let $S=$ $\left(x_{1}, \ldots, x_{K}\right)$ be a finite set of points in $M_{0}$ and let $0<r_{0}<R_{0}$ be real numbers such that $2 r_{0}<R_{0}$ and

(i) $B\left(x_{i}, r_{0}\right) \subseteq$ Int $M_{0}$,

(ii) $\left\{B\left(x_{i}, R_{0}\right)\right\}$ cover $M_{0}$,

(iii) $R_{0}<$ the injectivity radius of $M$,

(iv) no point of $M_{0}$ can be in more than $N$ of sets $B\left(x_{i}, R_{0}\right)$.

Set $M_{1}=M_{0}-\bigcup_{i=1}^{K} B\left(x_{i}, r_{0}\right)$.

Then we can find a constant $c>0$ which depends only on $(M, g)$ such that

$$
\lambda\left(M_{1}\right) \geqslant\left\{\begin{array}{l}
\frac{c K r_{0}^{m-2}}{N d \operatorname{vol}\left(M_{0}\right)} \quad \text { if } m=\operatorname{dim} M>2, \\
\frac{c K}{N d \operatorname{vol}\left(M_{0}\right)\left|\log r_{0}\right|} \quad \text { if } \operatorname{dim} M=2,
\end{array}\right.
$$

where $d$ is a number such that $K R_{0}^{m}=d \operatorname{vol}\left(M_{0}\right)$.

NotE. The expression "crushed ice" for this sort of situation is due to Rauch and Taylor [8], [9], where they view the $B\left(x_{i}, r_{0}\right)$ as $K$ little coolers (round ice cubes) and $\lambda$ represents the "rate" of cooling. This part of the proof is essentially theirs, our contribution being the refined "even spacing" lemma. 
Proof. Let $\psi: M_{1} \rightarrow \mathbf{R}$ be such that $\psi \mid \partial M_{1}=0$ and $\Delta \psi=\lambda\left(M_{1}\right) \psi$. Let $A_{i}=\{x$ $\left.\in M \mid r_{0} \leqslant d\left(x, x_{i}\right)<R_{0}\right\}$ and let $M_{2}=\cup A_{i}$. Extend $\psi$ to a continuous function with square integrable weak differential on $M_{2}$, by making it zero on $M_{2}-M_{1}$. Denote this also by $\psi$.

$$
\int_{M_{1}}|\operatorname{grad} \psi|^{2}=\int_{M_{2}}|\operatorname{grad} \psi|^{2} \geqslant \frac{1}{N} \sum_{i} \int_{A_{i}}|\operatorname{grad} \psi|^{2} .
$$

If on the set $A_{i}$ we introduce Riemann normal coordinates centered at $x_{i}$, and if $r$ is the radial coordinate, we see

$$
\int_{A_{i}}|\operatorname{grad} \psi|^{2} \geqslant \int_{A_{i}}\left(\frac{\partial \psi}{\partial r}\right)^{2} d v
$$

Let $V=\left\{v \in T M \mid\|v\| \leqslant R_{0}\right\}$ and let $E: \quad V \rightarrow M \times M$ be $E(v)=(p(v)$, $\left.\exp _{p(v)} v\right)$, where $p$ is the bundle projection. If $d v_{E}$ is the Euclidean volume element on the tangent spaces and if $d v$ is the Riemannian volume element, then

$$
E^{*}(d v(E(x, v)))=f(x, v) d v_{E}, \quad v \in T_{x} M .
$$

Thus $f$ is a smooth positive function defined on a compact set and we can find some number $c>0$ which depends on $(M, g)$ alone such that

$$
\int_{A_{i}}|\operatorname{grad} \psi|^{2} \geqslant c \int_{A_{i}}\left(\frac{\partial \psi}{\partial r}\right)^{2} r^{m-1} d r d \Sigma,
$$

where $d \Sigma$ is the volume element of the unit $m-1$ sphere in $\mathbf{R}^{m}$. We can then apply the argument in $[9$, p. 45$]$ to get the estimate

$$
\int_{A_{i}}|\operatorname{grad} \psi|^{2} \geqslant c^{\prime}\left(\int_{A_{i}} \psi^{2} d v\right) \frac{r_{0}^{m-2}}{R_{0}^{m}} \text { for } m>2,
$$

and a slightly different estimate involving logs for $m=2$. Therefore,

$$
\int_{M_{1}}|\operatorname{grad} \psi|^{2} \geqslant \frac{c^{\prime}}{N} \frac{r_{0}^{m-2}}{R_{0}^{m}} \sum_{i} \int_{A_{i}} \psi^{2} \geqslant \frac{c^{\prime} K r_{0}^{m-2}}{R_{0}^{m} K} \int_{M_{1}} \psi^{2},
$$

and hence, $\lambda\left(M_{1}\right) \geqslant c^{\prime} r_{0}^{m-2} / N d \operatorname{vol}\left(M_{0}\right)$ because $R_{0}^{m} K=d \operatorname{vol}\left(M_{0}\right)$. The case $m=2$ follows in a similar way.

Proposition 2.1 is our main analytic lemma; we now need the even spacing notions.

Definition 2.2. Let $(M, g)$ be a Riemannian manifold possibly with boundary and let $d$ be the induced metric. We say a sequence of finite subsets of points $S_{n}=\left\{x_{1}, \ldots, x_{K_{n}}\right\}, x_{i} \in$ Int $M$, are evenly spaced if for each $n$ we can find a number $R(n)$ such that the balls $B\left(x_{i}, R(n)\right), 1<i<K_{n}$, cover $M$ and such that we can find a number $N$ independent of $n$, such that each point of $M$ is in no more than $N$ of the $B\left(x_{i}, R(n)\right)$ for each $n$.

Theorem 2.3 (Even Spacing Lemma). Let $M$ be a compact connected m-dimensional Riemannian manifold and let $M_{0} \subseteq M$ be a compact connected m-dimensional submanifold with boundary $\partial M_{0}=N$. Then we can find sets of points $S_{n}=$ $\left\{x_{1}, \ldots, x_{K_{n}}\right\}$ in $M_{0}$ which are evenly spaced in $M_{0}$. Let $R(n)$ be the radius in the 
definition. We can also find numbers $r(n)>0, r(n)<R(n) / 2$ such that the sets $B\left(x_{i}, r(n)\right)$ satisfy

(a) $B\left(x_{i}, r(n)\right) \subseteq \operatorname{Int}\left(M_{0}\right), 1 \leqslant i \leqslant K_{n}$,

(b) $B\left(x_{i}, r(n)\right)$ and $B\left(x_{i}, R(n)\right)$ are diffeomorphic to smooth Euclidean balls,

(c) $B\left(x_{i}, r(n)\right)$ are pairwise disjoint,

(d) $K_{n}=K_{0} 2^{m n}, R(n)=R_{0} / 2^{n}$ and $r(n)=r_{0} / 2^{n}$.

We can now apply the Even Spacing Lemma to Proposition 2.1. We can assume the number $R_{0}$ in 2.3 is less than the injectivity radius of $M$. Let $M_{n}=M_{0}-$ $\cup_{i=1}^{K_{n}} B\left(x_{i}, r(n)\right)$ and we see

$$
\lambda\left(M_{n}\right) \geqslant \begin{cases}\frac{C K_{n} r(n)^{m-2}}{N d_{n} \operatorname{vol}\left(M_{0}\right)}, & m>2, \\ \frac{C}{N d_{n} \operatorname{vol}\left(M_{0}\right)} \cdot \frac{K_{n}}{|\log r(n)|},\end{cases}
$$

where $d_{n} \operatorname{vol}\left(M_{0}\right)=R(n)^{m} K_{n}=K_{0} 2^{m n} R_{0}^{m} / 2^{m n}=K_{0} R_{0}^{m}=d_{0} \operatorname{vol}\left(M_{0}\right)$ and is independent of $n$. Hence $\lambda\left(M_{n}\right) \rightarrow \infty$ as $n \rightarrow \infty$ and we get the following theorem.

TheOReM 2.4. Let $M, M_{0}$ and $\partial M_{0}=N$ be as above. Then for each number $K>0$ we can find $r_{0}>0$ and a finite set of points $x_{1}, \ldots, x_{s}, x_{i} \in M_{0}$, such that

(i) $B\left(x_{i}, r_{0}\right) \subseteq$ Int $M_{0}$;

(ii) the $B\left(x_{i}, r_{0}\right)$ are pairwise disjoint;

(iii) if $M_{1}=M_{0}-\cup_{i=1}^{s} B\left(x_{i}, r_{0}\right)$ then $\lambda\left(M_{1}\right)>K$.

REMARK. This discussion also holds for the case where we have Neumann boundary data on $N=\partial M_{0}$ and zero boundary data on the boundaries of the $B\left(x_{i}, r_{0}\right)$. One follows [8], [9] as we just did and uses the Lions' reflection to extend $\psi$ from $M_{0}$ to all of $M$ without significantly changing its $H_{1}$ norm.

\section{COROLlaRY 2.5. Theorem A for the Laplacian follows from Theorem 2.4.}

Proof. Pick $K>0$. Let us apply Theorem 2.4 and find a finite number of points $x_{1}, \ldots, x_{s}$ and a number $r>0, r<$ injectivity radius of $M$ such that the geodesic balls $B\left(x_{i}, r\right)=B_{i}, \quad 1 \leqslant i \leqslant s$, are pairwise disjoint in Int $M_{0}$ and $\lambda\left(M_{0}-\right.$ $\left.\cup B\left(x_{i}, r\right)\right)>K$. For each $B_{i}$ let $\gamma_{i}$ be a smooth curve joining $\partial B_{i}$ to $\partial M_{0}$, which meets these boundaries perpendicularly. Thicken $\gamma_{i}$ a bit and smooth the edges at the boundaries to form a tube denoted by $\tau_{i}$. Let $O_{i}=B_{i} \cup \tau_{i}$ and we can assume we have chosen the $\gamma_{i}$ and $\tau_{i}$ such that the $O_{i}$ are pairwise disjoint. Then $\lambda\left(M_{0}-\right.$ $\left.\cup O_{i}\right) \geqslant \lambda\left(M_{0}-\cup B_{i}\right) \geqslant K$. We can now define our diffeomorphism $h_{t}$ by eliminating the $O_{i}$ 's one at a time, by first shrinking the ball and then withdrawing the tube as in the pictures. 

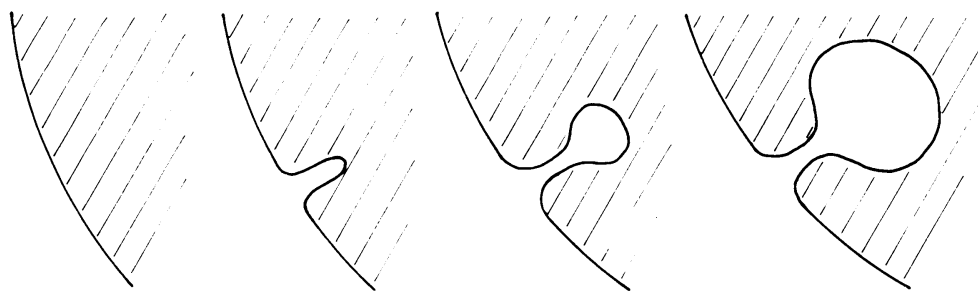

3. The proof of Theorem 2.3 (the Even Spacing Lemma). We will first discuss the case where $M_{0}=M$ and $\partial M_{0}=\varnothing$. We will then show how to alter the proof for the general case.

Let us isometrically embed $M$ in $\mathbf{R}^{s}$ for $s$ sufficiently large. Let $\pi: U_{\eta} \rightarrow M$ be the tubular neighborhood of radius $\eta$ of $M$ in $\mathbf{R}^{s}\left(U_{\eta}=\left(x \in \mathbf{R}^{s} \mid\|x-M\| \leqslant \eta\right.\right.$ and $\pi$ is the projection back onto $M)$ ). Following Whitney [12], for any $\eta>0$ we can find a simplicial complex $K, K \subseteq U_{\eta}$ (geometrically in $\mathbf{R}^{s}$, not just abstract) with the following properties.

(a) $K$ is itself a topological $m$-dimensional manifold.

(b) $\pi \mid K: K \rightarrow M$ is a homeomorphism of $K$ onto $M$.

(c) Each $m$-simplex $\sigma \subseteq K$ is almost parallel to the tangent plane $T_{\pi(x)}(M)$ for each $x \in \sigma$. More precisely for each $m$-simplex $\sigma \subseteq K$, there exists a constant $c>0$, such that $\left|\operatorname{det}\left(T_{\pi} \mid \sigma\right)\right| \geqslant c>0$.

The locally affine structure in $K$ allows us to speak of a distance $d_{L}$ between two points (the inf of the arclengths of continuous piecewise affine paths joining two points) and an $m$-volume $v_{L}$ of measurable subsets of $K$. Let $d$ and $v$ be the corresponding distance and volume defined by the Riemannian structure on $M$. It is easy to see from the properties of our triangulation and the compactness of $M$ that we can find positive numbers $c_{1}, c_{2}, d_{1}$ and $d_{2}$ such that

(A) for $x, y \in K, c_{1} d(\pi(x), \pi(y)) \leqslant d_{L}(x, y) \leqslant c_{2} d(\pi(x), \pi(y))$;

(B) for any measurable $S \subseteq K, d_{1} v(\pi(S)) \leqslant v_{L}(S) \leqslant d_{2} v(\pi(S))$.

Let $\sigma$ be an $m$-simplex of $K, \sigma=p_{0}, \ldots, p_{m}$. Set $v_{i}=p_{i}-p_{0}$. Then $\sigma$ is congruent to an $m$-simplex with 0 as a vertex and $v_{1}, \ldots, v_{m}$ as edges. For each $\sigma$ we can then form $2^{m}-1$ other $m$-simplices $\sigma\left(i_{1}, \ldots, i_{m}\right)$ where 0 is a vertex and the edges are $i_{j} v_{j}, i_{j}= \pm 1 \quad(\sigma \rightleftarrows \sigma(1, \ldots, 1))$.

In each $\sigma \subseteq K$, there is a point $x_{\sigma} \in \sigma$ which is the center of the largest inscribed ball $B_{L}\left(x_{\sigma}, r_{\sigma}\right)$ of radius $r(\sigma)$. Let $r_{\sigma}\left(i, \ldots, i_{m}\right)$ be the radius of the corresponding inscribed ball in $\sigma\left(i_{1}, \ldots, i_{m}\right)$, and set

$$
\tilde{r}_{0}<\inf _{\sigma}\left(r_{\sigma}\left(i_{1}, \ldots, i_{n}\right) / 2\right) \text {. }
$$

Let $p_{\sigma}=\pi\left(x_{\sigma}\right)$, pick $r_{0}<\tilde{r}_{0} / c_{2}$ and note that the ball

$$
B\left(p_{\sigma}, r_{0}\right)=\left\{y \mid d\left(y, p_{\sigma}\right)<r_{0}\right\} \leqslant \pi\left(B_{L}\left(x_{\sigma}, \tilde{r}_{0}\right)\right) .
$$

Hence the balls $B\left(p_{\sigma}, r_{0}\right)$ are disjoint.

Let $K_{1}$ be the standard subdivision of $K$. For all the properties of $K_{1}$ and its definition see [12]; we will need the following facts. Each $m$-simplex $\sigma$ is subdivided into $2^{m} m$-simplices of $K_{1}$. Furthermore if $\sigma=p_{0}, \ldots, p_{m}$ is an $m$-simplex of $K$, 
form the congruent $m$-simplex with edges $v_{i}=p_{i}-p_{0}$ and a vertex at 0 . Then each of the $m$-simplices $\sigma^{1}$ of $K_{1}$ into which $\sigma$ has been subdivided is congruent to one of the simplices with a vertex 0 and edges $i_{j} v_{j}, i_{j}= \pm \frac{1}{2}$. Thus if $x_{\sigma(1)}$ are the centers of the inscribed balls of the $m$-simplices $\sigma(1)$ of $K_{1}$, then the balls $B_{L}\left(x_{\sigma(1)}, \tilde{r}_{0} / 2\right)$ are disjoint, as are

$$
B\left(p_{\sigma(1)}, r_{0} / 2\right)=\left\{y \in M \mid d\left(p_{\sigma(1)}, y\right)<r_{0} / 2\right\} \quad \text { where } p_{\sigma(1)}=\pi\left(x_{\sigma(1)}\right) .
$$

Let $R_{\sigma}$ be the radius of the ball centered at $x_{\sigma}$ which contains $\sigma, \sigma \subseteq K$ on $m$-simplex and let $R_{\sigma}\left(i_{1}, \ldots, i_{m}\right)$ be the corresponding radius for the simplex $\sigma\left(i_{1}, \ldots, i_{m}\right)$. Set $\tilde{R}_{0}=\sup R_{\sigma}\left(i, \ldots, i_{n}\right)$. The balls $B_{L}\left(p_{\sigma}, \tilde{R}_{0}\right)$ cover $K$ and if we choose $R_{0} \geqslant c_{1} \tilde{R}_{0}$ and $R_{0}>2 r_{0}$, we see that the balls $B\left(p_{o}, R_{0}\right)$ cover $M$. Let us pass to the standard subdivision $K_{1}$. If $\sigma(1)$ is an $m$-simplex in $K_{1}$, then the balls $B\left(p_{\sigma(1)}, R_{0} / 2\right)$ must cover $K$.

Let $\tilde{\delta}_{0}$ be the supremum of the diameters of $m$-simplices $\sigma\left(i_{1}, \ldots, i_{m}\right)$ as $\sigma$ runs through the $m$-simplices of $K$. Let $\delta_{0}=\tilde{\delta}_{0} / c_{1}$. Hence diameter $(\pi(\sigma))<\delta_{0}$.

If we replace $K$ by $K_{1}$, then $\tilde{\delta}_{0}$ and $\delta_{0}$ are replaced by $\tilde{\delta}_{0} / 2$ and $\delta_{0} / 2$. Let $d(p)=$ distance from $p$ to its first cut point and let $\gamma=\min d(p)$. Replacing $K$ by $K_{(n)}$ ( $n$th standard subdivision) if need be, we can assume $2 R_{0}+\delta_{0}<\gamma$.

Let us collect what we have been able to show. Let us pick $K$ as above, $r_{0}$ and $R_{0}$ such that $r_{0}<2 R_{0}$ and $2 R_{0}+\delta_{0}<\gamma$. Let $K_{(n)}$ be the $n$th standard subdivision of $K$. Let $\{\sigma(n)\}$ be the set of $m$-simplices of $K_{(n)}$. Let $\{x(\sigma(n))\}$ be the "centers" of the $\{\sigma(n)\}$ as constructed above and let $p(\sigma(n))=\pi(x(\sigma(n)))$. If we set $S_{n}=$ $\{(p(\sigma(n)))\}, r(n)=r_{0} / 2^{n}$ and $R(n)=R_{0} / 2^{n}$, we see that

(i) the balls $B(p(\sigma(n)), R(n))$ cover $M$;

(ii) the balls $B(p(\sigma(n)), r(n))$ are disjoint;

(iii) these balls are diffeomorphic to smooth euclidean balls, and

(iv) $r(n)<R(n) / 2$.

If $L(n)$ is the number of points in $S_{n}$, we then see that $L(n)=L(0) \cdot 2^{m n}$ and therefore

$$
L(n) r(n)^{m-2}=L(0) 2^{m n}\left(\frac{r_{0}^{m-2}}{2^{n}}\right)^{m-2}=\frac{L(0) r_{0}^{m-2}}{4^{n}} .
$$

Thus it remains to show the even spacing. Let $N_{\sigma}=$ the number of $p_{\sigma}$ in $B\left(p_{\sigma}, 2 R_{0}\right)$. Let $N_{0}=\sup _{\sigma \subseteq K} N_{\sigma}$ where the $\sigma$ 's are $m$-simplices. Then $N_{0}>$ largest number of sets $B\left(p_{a}, R_{0}\right)$ any point in $M$ may touch. We wish to estimate $N_{0}$. We know if $p_{\sigma^{\prime}} \in B\left(p_{\sigma}, 2 R_{0}\right)$ then $\pi\left(\sigma^{\prime}\right) \subseteq B\left(p_{\sigma}, 2 R_{0}+\delta_{0}\right)$. Hence

$$
v\left(B\left(p_{\sigma}, 2 R_{0}+\delta_{0}\right)\right)>\sum_{p_{o^{\prime}} \in B\left(p_{o}, 2 R_{0}\right)} v\left(\pi\left(\sigma^{\prime}\right)\right) .
$$

Let $\Sigma=\inf \left\{v_{L}\left(\sigma\left(i_{1}, \ldots, i_{m}\right)\right) \mid \sigma\right.$ an $m$-simplex $\left.\subseteq K\right\}$. We can find a constant $e$ depending on the metric $g$ above such that

$$
N_{0} \leqslant d_{2} e\left(2 R_{0}+\delta_{0}\right)^{m} / \Sigma^{\prime}
$$

If we replace $K$ by $K_{1}$ the standard subdivision, we are interested in the number $N_{1}$ of $p_{\sigma^{\prime}(1)}$ in a ball radius $R_{0}$ about a given $p_{\sigma(1)}$. Here $\delta_{0}$ is replaced by $\delta_{1}=\delta_{0} / 2 . d_{2}$ 
and $e$ remain unchanged. Each $m$-simplex $\sigma$ splits into $2^{m} m$-simplices of equal volume, so the number $\Sigma^{\prime}$ in the denominator is replaced by $\Sigma^{\prime} / 2^{m}$ and we see that

$$
N_{1} \leqslant \frac{d_{2} e\left(R_{0}+\delta_{0} / 2\right)^{m}}{(1 / 2)^{m} \Sigma^{\prime}}=N_{0},
$$

which completes our proof in the case where $M=M_{0}$.

Before we proceed to the case of a manifold with boundary we need a definition.

Definition. Let $x \in \partial M_{0}$, let $v \in T\left(M_{0}\right)_{x}$ be a unit vector perpendicular to $\partial M_{0}$ which points into $M_{0}$ and let $\gamma_{x}(t)(t \geqslant 0)$ be the unique geodesic, parametrized by arclength such that $\gamma_{x}(0)=x$ and $\dot{\gamma}_{x}(0)=v$. For small $t>0, \gamma_{x}[0, t] \subseteq M_{0}$ and $x$ is the unique point on $\partial M_{0}$ which is closest to $\gamma_{x}(t)$. Let $t_{x}$ be the supremum of all $t>0$ such that the above properties hold. Thus the paths $\gamma_{x}[0, t], t>t_{x}$, no longer minimize the distance from $\gamma_{x}(t)$ to $\partial M_{0}$. The point $\gamma_{x}\left(t_{x}\right)$ is called the focal cut point of $\partial M_{0}$ along $\gamma_{x}$ and the set of points $\left\{\gamma_{x}\left(t_{x}\right) \mid x \in \partial M_{0}\right\}$ is called the focal cut locus of $\partial M_{0}$.

We now proceed to the general case. Let $\delta_{0}$ and $\gamma$ be as before. Let $K$ be the complex. Let

$$
\begin{aligned}
& A_{n}=\left\{m \text {-simplices in } K_{n} \text { such that } \pi(\sigma) \subseteq \text { Int } M_{0}\right\}, \\
& B_{n}=\left\{m \text {-simplices in } K_{n} \text { such that } \pi(\sigma) \cap N=\partial M_{0} \neq \varnothing\right\}, \\
& C_{n}=\left\{m \text {-simplices in } K_{n} \text { such that } \pi(\sigma) \subseteq M-M_{0}\right\},
\end{aligned}
$$

where $K_{n}$ is the $n$th standard subdivision of $K$. We assume that our initial triangulation $K$ and our initial $R_{0}$ are so chosen that

(i) $R_{0}>3 \delta_{0}$

(ii) $2 R_{0}+\delta_{0}<\gamma$,

(iii) $A_{0}, B_{0}$ and $C_{0}$ are all nonempty,

(iv) $2 \delta_{0}<$ distance from $\partial M_{0}$ to its focal cut locus.

By successively subdividing $K$ if necessary we can clearly arrange this situation. Let $S_{n}=\left\{p_{\sigma(n)} \mid B\left(p_{\sigma(n)}, r(n)\right) \subseteq\right.$ Int $\left.M_{0}\right\}$. The only thing left to show is that $\{B(p(\sigma(n)), R(n))\}$ cover $M$. If $x \in \pi(\sigma(n)), \sigma(n) \in A_{n}$, then $x \in B(p(\sigma(n)), R(n))$. Say $x \in \pi(\sigma(b)) \cap M_{0}, \sigma(n) \in B_{n}$. Then we can find $y \in N$ such that $d(x, y)<$ $\delta_{0} / 2^{n}$. Let $T_{n}=\left\{z \in M_{0} \mid d\left(Z, 2 M_{0}\right)=\delta_{0} / 2^{n}\right\}$. It is easy to see that each $x \in T_{n}$ is in $\pi(\sigma(n))$ for some $\sigma(n) \in A_{n}$. Hence we can find some $\sigma(n) \in A_{n}$ such that $d(y, p(\sigma(n)))<2 \delta_{0} / 2^{n}$ and thus $d(x, p(\sigma(n)))<3 \delta_{n} / 2^{n}<R_{0} / 2^{n}$.

REMARK. A careful accounting of this construction shows the following result. For each $\varepsilon>0$ we can choose the original $r_{0}$ so small that $\operatorname{vol}\left(M_{0}-M_{n}\right)<\varepsilon$ for all $n$, where $M_{n}=M_{0}-\cup_{\sigma(n) \in K_{n}} B\left(x_{\sigma(n)}, r_{0} / 2^{n}\right)$.

\section{BIBLIOGRAPHY}

1. A. Baider, Non-compact Riemannian manifolds with discrete spectra, J. Differential Geometry (to appear).

2. M. Berger, P. Gauduchon and E. Mazet, Le spectre d'une variété Riemannienne, Lecture Notes in Math., vol. 194, Springer-Verlag, Berlin and New York, 1971.

3. I. Chavel and E. Feldman, Spectra of domains, J. Functional Analysis 30 (1978), 198-222.

4. S. Y. Cheng, Eigenfunctions and nodal sets, Comment. Math. Helv. 51 (1976), 43-55.

5. R. Courant and D. Hilbert, Methods of mathematical physics, vol. I, Interscience, New York, 1952. 
6. P. Hartman and C. R. Putnam, The least cluster point of the spectrum of boundary value problems, Amer. J. Math. 70 (1968), 849-855.

7. J. Piepenbrink, Nonoscillatory elliptic equations, J. Differential Equations 15 (1974), 541-550.

8. J. Rauch, Partial differential equations and related topics, Lecture Notes in Math., vol. 446, Springer-Verlag, Berlin and New York, 1975, pp. 354-389.

9. J. Rauch and M. Taylor, Potential and scattering theory on wildly perturbed domains, J. Functional Analysis 18 (1975), 27-59.

10. F. Riesz and B. Nagy, Functional analysis, Ungar, New York, 1955.

11. D. Stone, Geodesics in piecewise manifolds, Trans. Amer. Math. Soc. 215 (1976), 1-44.

12. H. Whitney, Geometric integration theory, Princeton Mathematical Series, vol. 21, Princeton Univ. Press, Princeton, N. J., 1957.

Department of Mathematics, Hunter College (CUNY), New York, New York 10021

Department of Mathematics, Graduate School (CUNY), New York, New York 10036 